\title{
WZY-321, a novel evodiamine analog, inhibits glioma cell growth in an autophagy-associated manner
}

\author{
GUAN SUN ${ }^{*}$, CHUANG ZHANG ${ }^{2 *}$, HONGMAO SONG ${ }^{3 *}, \mathrm{JUN} \mathrm{GUO}^{1}, \mathrm{MIN} \mathrm{LI}^{4}$ and YING CAO $^{3}$ \\ ${ }^{1}$ Department of Neurosurgery, The First People's Hospital of Yancheng, Fourth Affiliated Hospital of Nantong University, \\ Yancheng, Jiangsu 224001; ${ }^{2}$ Department of Medical Oncology, The Eighty-First Hospital of People's Liberation Army, \\ Nanjing, Jiangsu 210002; ${ }^{3}$ Department of Ear-Nose-Throat, Huai'an Second People's Hospital, \\ The Affiliated Huai'an Hospital of Xuzhou Medical University, Huai'an, Jiangsu 223002; ${ }^{4}$ Department of Neurosurgery, \\ Jiangning Hospital Affiliated with Nanjing Medical University, Nanjing, Jiangsu 211100, P.R. China
}

Received December 7, 2017; Accepted September 21, 2018

\section{DOI: $10.3892 / 01.2018 .9847$}

\begin{abstract}
Glioblastoma is one of the most aggressive types of brain tumor. The median survival rate of patients with glioblastoma (World Health Organization grade IV) is $<15$ months. Therefore, there is an urgent requirement for the development of novel and efficient therapeutic agents against glioma. In previous studies, WZY-321 (10-hydroxy-1-methyl-8,13b-dihydr o-5H,7H-benzo[e]benzofuro[2',3':3,4]pyrido[2,1- $b][1,3]$ oxazin5-one), a novel evodiamine (Evo) analog, was reported to exhibit enhanced pharmacological properties and improved cytotoxicity against a number of human cancer cell lines compared with Evo. In the current study, the anti-proliferative effect of WZY-321 on SHG-44 and SWO-38 glioma cells was further studied, and its mechanism of action investigated. The results indicated that WZY-321 inhibited the proliferation of SHG-44 cells in a dose- and time-dependent manner by enhancing cellular apoptosis and inducing cell cycle arrest at the G2-M phase. Treatment of glioma cells with WZY-321 concomitantly increased the expression levels of microtubule associated protein 1 light chain $3 \alpha$ and Beclin1, indicating enhanced autophagy. Overall, the results of the present study revealed the anti-proliferative potential of WZY-321 in glioma cells, thus providing a possible autophagy-based therapeutic strategy for the treatment of glioblastoma.
\end{abstract}

Correspondence to: Dr Ying Cao, Department of Ear-Nose-Throat, Huai'an Second People's Hospital, The Affiliated Huai'an Hospital of Xuzhou Medical University, 387 Fujian Road, Huai'an, Jiangsu 223002, P.R. China

E-mail: cy20101462@163.com

Dr Min Li, Department of Neurosurgery, Jiangning Hospital Affiliated with Nanjing Medical University, 688 Wanan Road, Nanjing, Jiangsu 211100, P.R. China

E-mail: sbn133@163.com

*Contributed equally

Key words: evodiamine, autophagy, apoptosis, beclin1, glioma

\section{Introduction}

The prognosis of patients with glioblastoma multiforme (GBM) and anaplastic astrocytoma remains poor, despite the current treatment modalities (1). Generally, radiation and chemotherapy are initiated following surgical treatment to improve the curative effects (2). Temozolomide (TMZ), a chemotherapeutic drug, is used as the drug of choice for treating malignant gliomas in clinical practice. The cytotoxicity of TMZ is associated with its ability to methylate guanine at the $\mathrm{O}^{6}$ position (3). Although TMZ is used as a second- and first-line treatment for astrocytoma and GBM, respectively, its effect is barely satisfactory as it extends the median survival of patients by only 2 months (4-6). Cells containing elevated levels of $\mathrm{O}^{6}$-methylguanine DNA methyltransferase repair the aberrant methylation induced by TMZ, and consequently reverse the cytotoxic effect. In addition, DNA mismatch repair deficiency makes cells tolerant to methylation and to the cytotoxic effects of TMZ (7). Therefore, the development of novel chemotherapeutic drugs for the treatment of gliomas is essential.

Evading apoptosis is a major hallmark of tumorigenesis and chemoresistance, particularly in high-grade glioblastomas and astrocytomas (8). Recently, particular attention has been paid to the crosstalk between apoptosis and autophagy. The term 'autophagy,' which originates from a Greek term meaning self (auto)-eating (phagy), was first coined and defined by De Duve in 1963 (9). Autophagy is the degradation process of cytoplasmic constituents in the lysosome-vacuole and is a fundamental mechanism, well conserved among eukaryotes (10). Autophagy serves a vital role in development, protein and organelle quality control, senescence, neurodegeneration and tumorigenesis. In 1999, Liang et al (11) first reported the role of Beclin1, one of the most important constituents for autophagosome formation, in the induction of autophagy and inhibition of tumorigenesis. In 2000, Kabeya et al (12) identified two forms of microtubule associated protein 1 light chain $3 \alpha$ (LC3), termed LC3-I and LC3-II, respectively. LC3-II serves an important role in the formation of the autophagosome membrane. Subsequent investigations in mammals used Beclin1 and 
LC3 as autophagic markers. In addition, Beclin1 and LC3 are reliable markers of brain tumors.

Autophagy is associated with drug resistance in numerous types of tumor cells. Notably, although autophagy impedes the therapeutic effects of anti-cancer drugs in some cases (13), it potentiates responses to conventional therapies in gliomas $(14,15)$. In addition, expression levels of the autophagic proteins Beclin1 and LC3-II are much lower in higher-grade astrocytomas compared with lower-grade astrocytomas and normal brain tissue, and prognosis is positively associated with the level of autophagy $(16,17)$. This evidence suggests that a decrease in autophagic activity may be involved in the progression of astrocytic or glioma tumors. Therefore, restoration of autophagy may inhibit tumor progression and may prove promising as a future therapeutic strategy.

Evodiamine (Evo) is a quinazolinocarboline alkaloid isolated from the fruit of Evodiae fructus, a traditional Chinese herb. E. fructus has been widely used for the treatment of gastrointestinal disorders, headache and postpartum hemorrhage (18). Evo has been reported to have various therapeutic benefits associated with the treatment of cancer, inflammation, obesity, cardiovascular diseases and pain (19-22). Studies investigating the anticancer activity of Evo have demonstrated that it inhibits the growth and metastasis of various cancer cells by regulating the cell cycle, apoptosis and autophagy $(21,23)$. Evo induces intracellular calcium-JNK signaling-mediated autophagy and calcium-mitochondria-mediated apoptosis in glioma cells (20). However, the potential of Evo is hindered due to its limited efficiency and drug-like properties, including aqueous solubility and rapid plasma clearance (24). The development of Evo analogs with optimized drug properties is crucial for the treatment of malignant gliomas. WZY-321 is a novel Evo analog with improved drug properties. WZY-321 has demonstrated promising cytotoxic effects in cancer cells (25). The present study evaluated the regulation of autophagy and the therapeutic potential of WZY-321 in gliomas.

\section{Materials and methods}

Reagents. The Cell Counting Kit-8 (CCK-8) kit and 3-methyladenine (3-MA) were purchased from Sigma-Aldrich (Merck KGaA, Darmstadt, Germany). Dulbecco's modified Eagle's medium (DMEM), fetal bovine serum (FBS) and penicillin-streptomycin were purchased from Gibco (Thermo Fisher Scientific, Inc., Waltham, MA, USA). The Annexin V-fluorescein isothiocyanate (FITC) apoptosis staining kit and antibodies used in the study were purchased from Thermo Fisher Scientific, Inc. The lysis buffer (RABLYSIS1) and propidium iodide (PI; cat. no. P4170) were purchased from Sigma-Aldrich (Merck KGaA). WZY-321, a novel analog of Evo (Fig. 1), was designed via a scaffold hopping strategy. A series of newly-designed Evo analogs, including WZY-321, were synthesized in the laboratory of Dr Shengtao $\mathrm{Xu}$ at the China Pharmaceutical University (Nanjing, China) (25). The chemical structure of WZY-321 was characterized by ${ }^{1} \mathrm{H}$ nuclear magnetic resonance (NMR), ${ }^{13} \mathrm{C} \mathrm{NMR}$, and mass spectroscopy. The purity of the compound was determined by analytical high performance liquid chromatography and the biologically evaluated compound was determined to be $98 \%$ pure.

Cell culture. SHG-44 glioma cell line was purchased from the American Type Culture Collection (Manassas, VA, USA). The human glioma cell line SWO-38 was established by the Department of Neurosurgery at the Jiangning Hospital (Nanjing, China) (26). The cells were cultured at $37^{\circ} \mathrm{C}$ in DMEM supplemented with $10 \%$ FBS, $200 \mathrm{mM}$ L-glutamine, $100 \mathrm{U}-\mathrm{ml}$ penicillin, $100 \mu \mathrm{g}-\mathrm{ml}$ streptomycin, $100 \mathrm{mM}$ sodium pyruvate and $1 \%$ non-essential amino acids. Cells were incubated at $37^{\circ} \mathrm{C}$ in a humidified incubator containing $5 \% \mathrm{CO}_{2}$. The medium was changed every 2 days and the cells were passaged every 3 days. The density of the seeded cells in different biological replicates was the same.

Assessment of cell viability. SHG-44 and SWO-38 cells $\left(5 \times 10^{3}\right.$ cells-100 $\left.\mu \mathrm{l}\right)$ were seeded into each well of a 96 -well plate and pre-incubated for $24 \mathrm{~h}$ in a humidified incubator $\left(37^{\circ} \mathrm{C} ; 5 \% \mathrm{CO}_{2}\right)$. Total volumes of $10 \mu \mathrm{l}$ of various concentrations of WZY-321 $(0,5,10,30$ and $50 \mu \mathrm{M})$ were added to the cells and incubated for 24 or $48 \mathrm{~h}$. Following incubation, $10 \mu$ l CCK-8 solution was added to each well and incubated for 1-4 h. Cell viability was proportional to the optical density and was quantitatively measured by spectrophotometry (Bio-Rad Laboratories, Inc., Hercules, CA, USA) at $450 \mathrm{~nm}$. The viability of cells in the control group incubated for $48 \mathrm{~h}$ was defined as $100 \%$ viable.

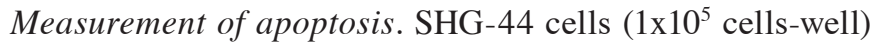
were cultured in complete medium in 6-well plates for $24 \mathrm{~h}$, and treated in triplicate with different concentrations of WZY-321 $(10,30$ and $50 \mu \mathrm{M})$ for $24 \mathrm{~h}$. The control cells were treated with vehicle [1\% dimethyl sulfoxide (DMSO) in complete medium]. Cell morphology was observed under optical microscope (magnification, x20). The cells were subsequently harvested, washed, and stained with PI and FITC-Annexin V in the dark at $25^{\circ} \mathrm{C}$ for 15 min using the Annexin V-FITC apoptosis staining kit, according to the manufacturer's protocols. The percentage of apoptotic cells was determined by flow cytometry using an FC500 cytometer (Beckman Coulter, Inc., Brea, CA, USA). Annexin V-FITC binding was analyzed by flow cytometry (excitation=488 nm; emission=350 nm) using the FITC signal detector (usually FL1), and PI staining by the phycoerythrin emission signal detector (usually FL2), using FlowJo 8.8 software (Tree Star, Inc., Ashland, OR, USA).

Cell cycle study. Progression through the cell cycle was assessed by flow cytometry DNA determination with PI. SHG-44 cells were seeded in 6 -well plates $\left(1 \times 10^{5}\right.$ cells-well) and incubated at $37^{\circ} \mathrm{C}$ for $24 \mathrm{~h}$. Cells were incubated with WZY-321 at certain concentrations $(0,10,30$ and $50 \mu \mathrm{M})$. Cells treated with the solvent (DMSO) were included. Following $24 \mathrm{~h}$ of treatment, cells were fixed with $70 \%$ ethanol at $-20^{\circ} \mathrm{C}$ for $12 \mathrm{~h}$, treated with RNase, and stained with PI at $37^{\circ} \mathrm{C}$ for 30 min. Cellular DNA content for the cell cycle distribution analysis was measured using a flow cytometer (FACSCalibur; BD Biosciences, Franklin Lakes, NJ, USA). The percentages of cells in different phases of cell cycle were analyzed by ModFit 4.1 software (Verity Software House, Topsham, ME, USA). 


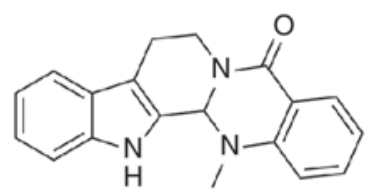

Evodiamine

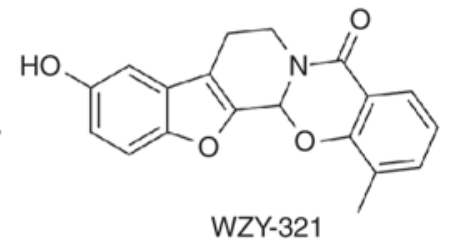

WZY-321
Figure 1. Chemical structure of evodiamine and its novel analog WZY-321.

Western blot analysis. SHG-44 cells were incubated at $37^{\circ} \mathrm{C}$ in the presence or absence of WZY-321 at a concentration of $0,10,30$ and $50 \mu \mathrm{M}$ for $24 \mathrm{~h}$. 3-MA was used as an autophagy inhibitor and cells were pretreated with $2.5 \mathrm{mM} \mathrm{3-MA}$ at $37^{\circ} \mathrm{C}$ for $12 \mathrm{~h}$ prior to treatment with WZY-321. Following incubation, cells were collected, centrifuged at 13,000 x g at $4^{\circ} \mathrm{C}$ for $15 \mathrm{~min}$ and washed twice with ice cold PBS. The pellets were re-suspended in lysis buffer. The cells were lysed on ice for $20 \mathrm{~min}$ and the lysates were centrifuged at $13,000 \mathrm{xg}$ at $4^{\circ} \mathrm{C}$ for $15 \mathrm{~min}$. The protein concentration in the supernatant was determined using bicinchoninic acid protein assay reagents. Equal amounts of protein (20 $\mu \mathrm{g}$-well) were separated via SDS-PAGE on a $10 \%$ gel and transferred to a polyvinylidene fluoride Hybond-P membrane. Membranes were blocked with 5\% non-fat milk for $1 \mathrm{~h}$ at room temperature and incubated with primary antibodies against: Beclin1 (cat. no. sc-48341); LC3 (cat. no. sc-271625); apoptosis regulator $\mathrm{Bcl}-2$ (Bcl-2; cat. no. sc-509); cyclin-dependent kinase 1 (cdc2; cat no. sc-54); cyclin D1 (cat. no. sc-70899); poly (ADP-ribose) polymerase (cat. no. sc-136208); and $\beta$-actin (cat. no. sc-58673) or GAPDH (cat. no. sc-293335) (1:1,000 dilution; all Santa Cruz Biotechnology, Inc., Dallas, TX, USA) by gentle rotation overnight at $4^{\circ} \mathrm{C}$. $\beta$-actin and GAPDH were used as loading controls. The membranes were subsequently washed and incubated with horseradish peroxidase (HRP)-conjugated secondary antibody (1:20,000 dilution; cat. no. sc-2489; Santa Cruz Biotechnology, Inc.) for $2 \mathrm{~h}$ at room temperature and visualized using enhanced chemiluminescent reagent (EMD Millipore, Billerica, MA, USA). The densitometric analysis was performed using Image J 1.48 software (National Institutes of Health, Bethesda, MD, USA).

Statistical analysis. Values are expressed as the mean \pm standard error of the mean calculated from three independent experiments. Statistical analysis was performed using Student's t-test (for two groups) or one-way analysis of variance followed by Duncan's multiple-range test (for three or more groups) Analysis was performed using GraphPad Prism 5 (GraphPad Software, Inc., La Jolla, CA, USA). $\mathrm{P}<0.05$ was considered to indicate a statistically significant difference.

\section{Results}

WZY-321 induces cytostatic effects in SHG-44 and SWO-38 glioma cells. SHG-44 and SWO-38 glioma (26) cells were pretreated with various concentrations of WZY-321 $(5,10$, 30 or $50 \mu \mathrm{M}$ ) for 24 or $48 \mathrm{~h}$. The number of viable cells was determined using the CCK-8 assay. As presented in Fig. 2, WZY-321 significantly reduced the viability of SHG-44 and
SWO-38 cells in a dose-dependent manner. Treatment with $10 \mu \mathrm{M}$ WZY-321 for $48 \mathrm{~h}$ reduced the cell count to $\sim 30 \%$ compared with the control group. In addition, incubation with WZY-321 for $24 \mathrm{~h}$ significantly decreased the viability of SHG-44 glioma cells. Prolonged incubation of cells with WZY-321 for up to $48 \mathrm{~h}$ displayed little benefit. SWO-38 cells were less sensitive to WZY-321 compared with SHG-44 cells. The histological subtypes of SHG-44 and SWO-38 cells may explain this sensitivity (27). Therefore, in the subsequent studies, SHG-44 glioma cells were incubated with WZY-321 for $24 \mathrm{~h}$ prior to processing the samples for subsequent pharmacological tests.

WZY-321 triggers apoptosis in SHG-44 glioma cells. SHG-44 cells treated with WZY-321 for $24 \mathrm{~h}$ displayed marked alterations in cell morphology, exhibiting chromatin condensation, which suggested the cytostatic effect of WZY-321 may be associated with cell apoptosis (Data not shown). To confirm whether WZY-321 is able to induce apoptosis in SHG-44 cells, vehicle- or WZY-321-treated SHG-44 cells were stained with Annexin V-PI for flow cytometry analysis. Externalization of phosphatidylserine (PS) from the inner leaflet to the outer leaflet of the plasma membrane is a distinct phenomenon observed in early apoptotic cells. Annexin V has high affinity for PS, and fluorochrome-labeled Annexin V may be used for the detection of apoptotic cells. By contrast, PI is used to detect necrotic cells due to its ability to permeate the damaged cell membrane. In the present study, flow cytometric analysis identified four groups of cells: i) Viable cells (Annexin V-, PI-); ii) early apoptotic cells (Annexin V+, PI-); iii) late apoptotic cells (Annexin $\mathrm{V}+, \mathrm{PI}+$ ); and iv) necrotic cells (Annexin V-, PI+). The percentage of apoptotic SHG-44 cells detected by Annexin V-FITC and PI double staining following incubation with various concentrations of WZY-321 $(0,10,30$ or $50 \mu \mathrm{M})$ for $24 \mathrm{~h}$ is presented in Fig. 3. The number of early-stage apoptotic SHG-44 cells was increased in the $50 \mu \mathrm{M}$ WYZ-321-treated group (11.3\%) compared with that in the control group (1.6\%), in a dose-dependent manner. The upper left quadrants representing the necrotic cells were comparable in each group. However, the increase in the number of advanced stage apoptotic cells following treatment with WZY-321 was not significant. These results demonstrated that WZY-321 may dose-dependently induce apoptosis in SHG-44 cancer cells.

WZY-321 induces cell cycle arrest at the G2-M phase. One of the mechanisms by which chemical antitumor agents inhibit cell proliferation is through the induction of cell cycle arrest (28). To determine this mechanism involved in the suppression of cell growth by WZY-321, the DNA content of the cell nuclei was detected by flow cytometry. SHG-44 cells were treated with varying concentrations of WZY-321 $(0,10$, 30 and $50 \mu \mathrm{M}$ ). As presented in Fig. 4A and B, the number of cells which arrested at the G2-M phase increased from 45.9 to $51.6 \%$ in a dose-dependent manner, along with a concomitant decrease in the percentage of cells in the S and G1 phases. Western blot analysis was performed to determine the expression levels of cell cycle regulatory proteins. As displayed in Fig. 4C, treatment of SHG-44 cells with WZY-321 resulted in a decrease in the protein expression levels of cdc 2 and 

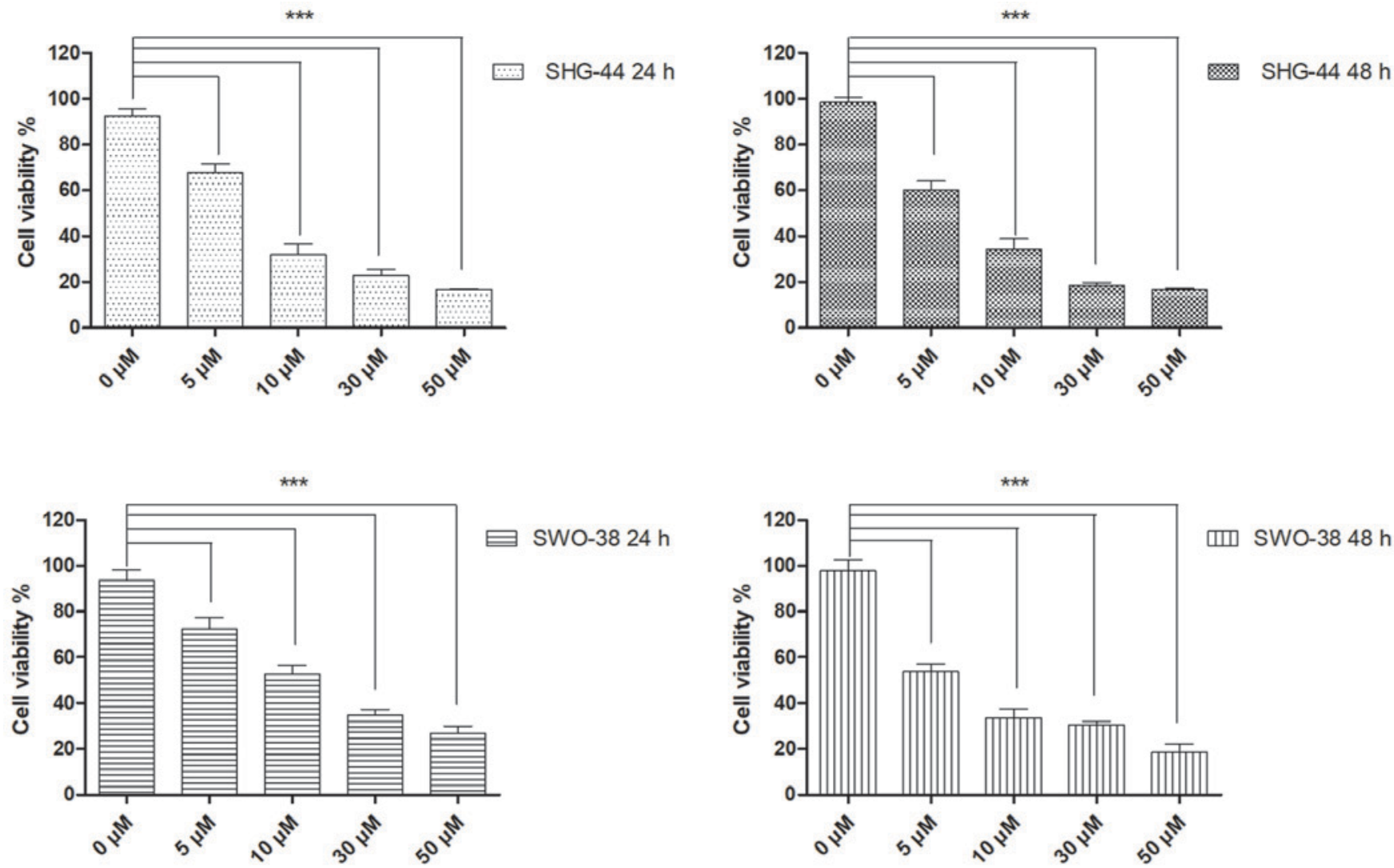

Figure 2. Effect of WZY-321 on the viability of SHG-44 and SWO-38 cells. SHG-44 and SWO-38 cells were treated with various concentrations of WZY-321 $(0,5,10,30$ and $50 \mu \mathrm{M})$ for 24 and $48 \mathrm{~h}$. Cell viability was measured using the Cell Counting Kit- 8 assay. Data presented are the mean \pm standard error of the mean of three independent experiments. ${ }^{* * *} \mathrm{P}<0.001$, control compared with treated cells.
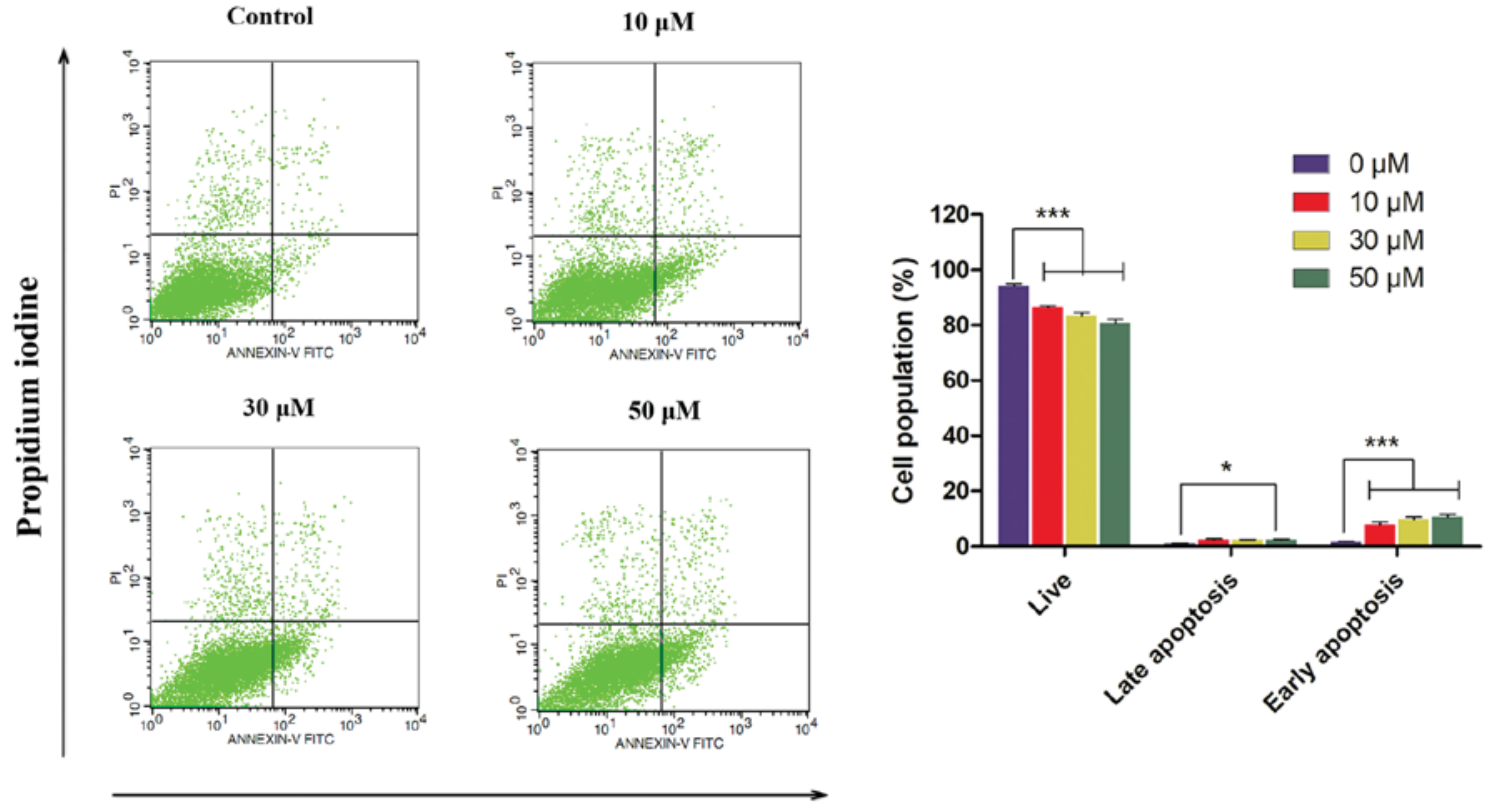

\section{Annexin V-FITC}

Figure 3. WZY-321 induces apoptosis in SHG-44 cells. SHG-44 cells were treated with varying concentrations of WZY-321 $(0,10,30$ and $50 \mu \mathrm{M})$ for $24 \mathrm{~h}$. Following incubation, cells were stained with Annexin V-PI and analyzed by flow cytometry. ${ }^{*} \mathrm{P}<0.05,{ }^{* * * *} \mathrm{P}<0.001$, control compared with treated cells. PI, propidium iodide; FITC, fluorescein isothiocyanate.

cyclin D1, in a dose-dependent manner. These results suggest an association between WZY-321-induced apoptosis and the accelerated entry of cells into G2-M arrest.
WZY-321 enhances autophagy in SHG-44 glioma cells. The cytotoxicity of certain anti-tumor agents is induced by autophagy (29-31). To assess the association of autophagy with the 

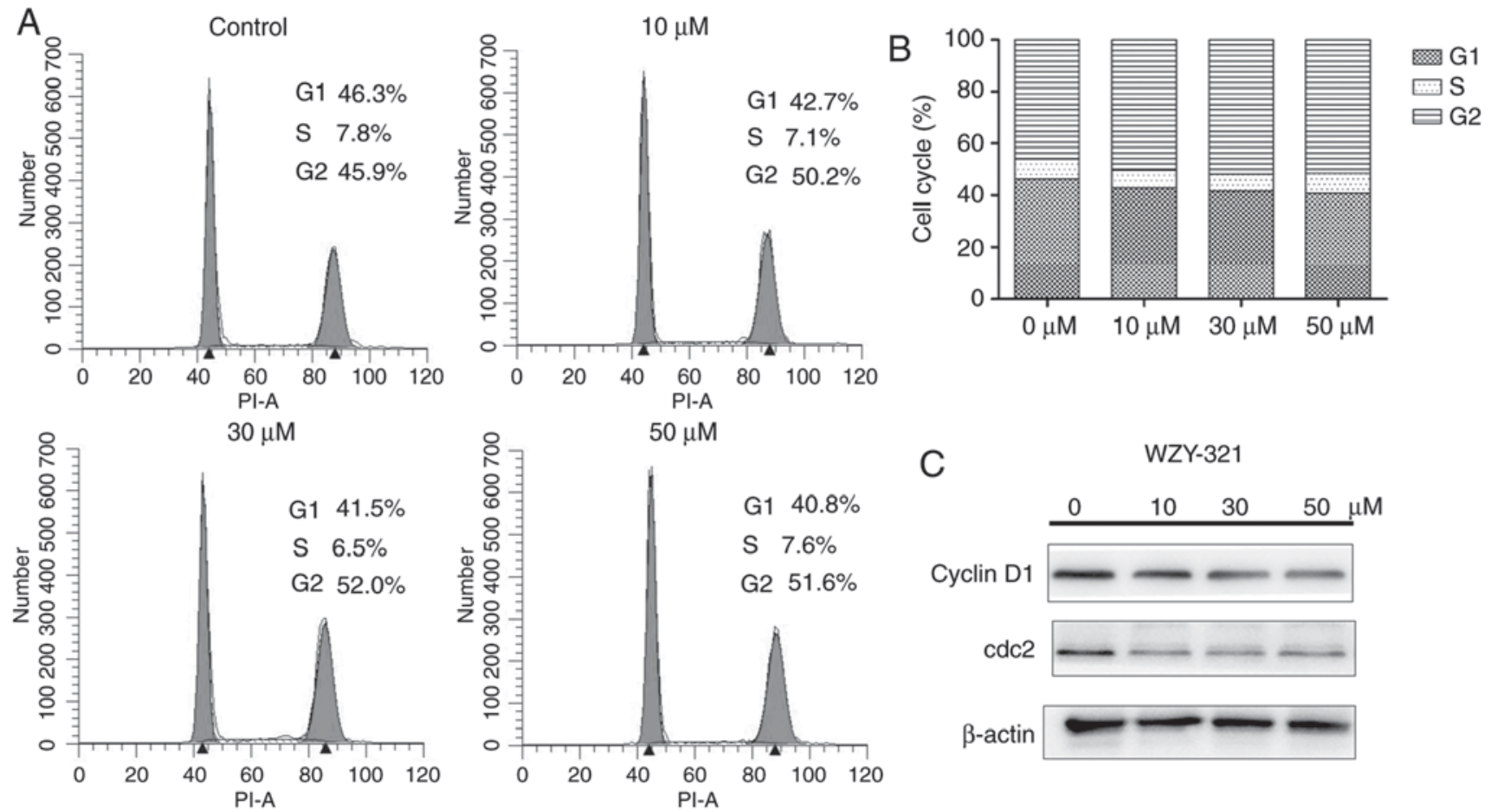

C

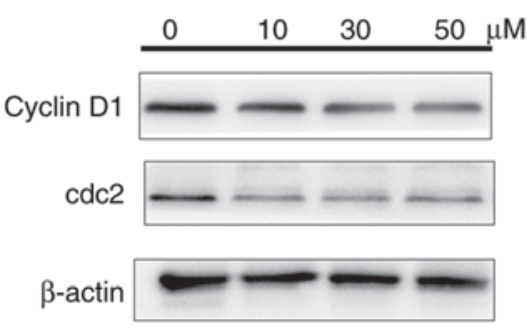

Figure 4. WZY-321 induces G2-M phase arrest in SHG-44 cells. (A) SHG-44 cells were treated with varying concentrations of WZY-321 (0, 10, 30 and $50 \mu \mathrm{M})$ for $24 \mathrm{~h}$. Cells were harvested, stained with PI and analyzed by flow cytometry. The percentages of cells in the G1, S and G2 phases of the cell cycle were calculated. (B) Histograms displaying the percentages of cells in the G1, S and G2 phases of the cell cycle. (C) Western blot analysis displaying the effect of WZY-321 on the expression levels of the G2-M regulatory proteins Cyclin D1 and cdc2. cdc2, cyclin-dependent kinase 1; PI, propidium iodide.

pro-apoptotic effects of WZY-321, a number of autophagic markers were analyzed. Beclin1, a novel Bcl-2-interacting coiled-coil protein has structural similarity to yeast autophagy-related protein 6 . The proven importance of Beclin1 in tumor suppression triggered research into the role of autophagy in tumor suppression. LC3 is the mammalian homolog of yeast autophagy-related gene 8. LC3-II is derived from LC3-I following cleavage from the C-terminal and serves an important role in the formation of autophagosomes, as it localizes to the outer and inner membranes. Beclin1 and LC3 are frequently-used markers for the evaluation of tumorigenesis in brain tumors. In the present study, SHG-44 glioma cells were incubated with varying concentrations of WZY-321 for $24 \mathrm{~h}$ and the expression levels of Beclin1 and LC3 were evaluated. As presented in Fig. 5, incubation with various concentrations of WZY-321 increased the expression levels of Beclin1 and LC3-II. Accordingly, statistical analysis of the immunoblotting confirmed the above findings (Fig. 5). Notably, while expression levels of Beclin1 and LC3-II in cells incubated with $30 \mu \mathrm{M}$ WZY-321 were comparable with those treated with $10 \mu \mathrm{M}$ WZY-321, levels in the $50 \mu \mathrm{M}$ group were slightly higher compared with the untreated group for LC3-II-LC3-I ("P<0.05), and significantly higher for Beclin- $1\left({ }^{* *} \mathrm{P}<0.01\right)$, indicating a dose-dependent induction of autophagy by WZY-321 in SHG-44 cells.

Treatment with an autophagy inhibitor inhibits the apoptotic effects of WZY-321. A complex association exists between autophagy and apoptosis. The activation of autophagy may potentiate cytotoxicity, or impede autophagic cell death. In order to probe whether enhanced autophagy contributed to the anti-cancer potential of WZY-321, or impeded the therapeutic effects of WZY-321, 3-MA was used as an autophagy inhibitor and the expression levels of Bcl-2 and Beclin1 were analyzed (Fig. 6). In WZY-321-treated SHG-44 cells, a marked decrease was observed in the protein expression of Bcl-2, confirming the apoptotic effect of WZY-321. However, this apoptotic effect was blocked when cells were pretreated with 3-MA prior to treatment with WZY-321. As presented in Fig. 6B, the expression level of Beclin1 was lower in 3-MA-pretreated SHG-44 cells treated with WZY-321 compared with cells treated with WZY-321 alone. In addition, the expression levels of Bcl-2 were higher in 3-MA-pretreated SHG-44 cells treated with WZY-321 compared with cells treated with WZY-321 alone.

\section{Discussion}

In the present study, it was hypothesized that there is an association between autophagy and the anti-tumorigenic potential of WZY-321. Treatment with WZY-321 inhibited the growth of glioma cells in a dose-dependent manner. This is the first time, to the best of our knowledge, that the association of the anti-tumorigenesis potential of WZY-321 with increased autophagic levels in glioma has been demonstrated.

Recent studies have reported that Evo inhibits the proliferation of a variety of cancer cells by inducing apoptosis $(20,32)$. However, the moderate anti-tumor potency, sub-optimal physicochemical properties and patent ineligibility of Evo have impeded its direct clinical application. Structural modification of natural products may increase their potential to be used as drugs, in addition to the likelihood of obtaining patents. WZY-321 is a novel analog of 

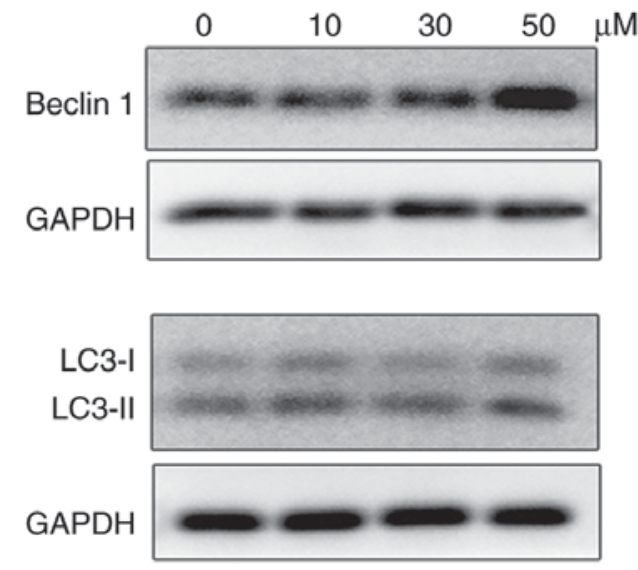
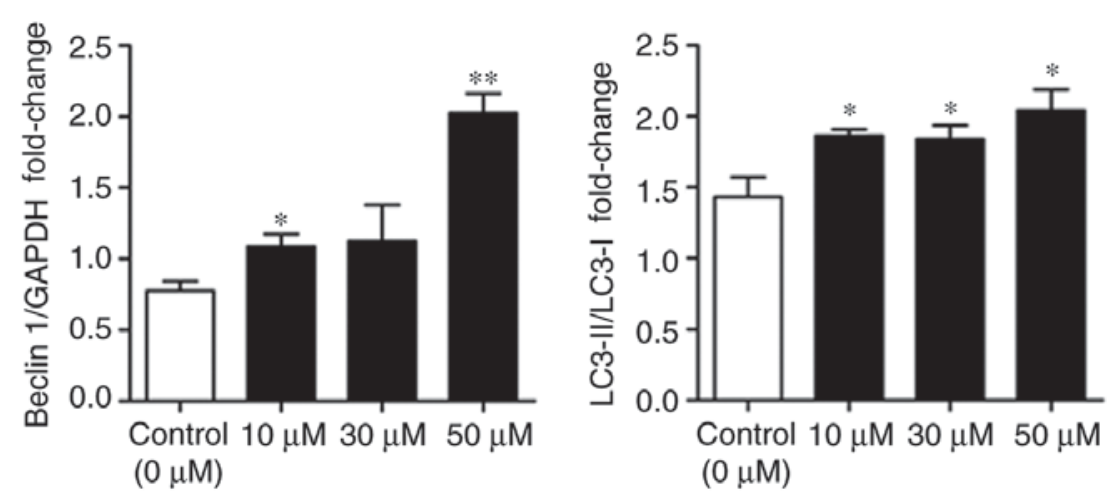

Figure 5. Effects of WZY-321 on autophagy. SHG-44 cells were treated with various concentrations of WZY-321 $(0,10,30$ and $50 \mu \mathrm{M})$ for $48 \mathrm{~h}$. Western blotting was performed using specific antibodies, to determine the expression levels of Beclin1 and LC3. GAPDH was used as the internal control. The density ratio of proteins to GAPDH is illustrated as relative expression. The histograms display the density ratios of LC3-II to LC3-I. Data represent the mean \pm standard error of the mean of three independent experiments. ${ }^{*} \mathrm{P}<0.05,{ }^{* *} \mathrm{P}<0.01$ vs. control. LC 3 , microtubule associated protein 1 light chain $3 \alpha$.
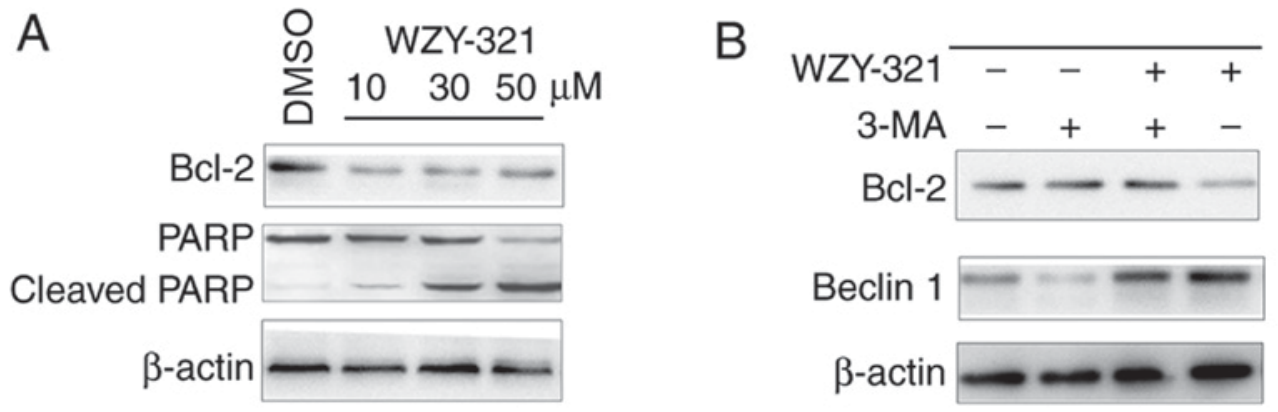

Figure 6. Role of autophagy in the anti-cancer potential of WZY-321. (A) HG-44 cells were treated with DMSO, or varying concentrations of WZY-321 (10, 30 and $50 \mu \mathrm{M}$ ). The expression levels of Bcl-2 and beclin1 were assessed by western blotting following $24 \mathrm{~h}$ of incubation. (B) SHG- 44 cells were treated with DMSO, WZY-321 $(30 \mu \mathrm{M})$ or 3-MA $(2.5 \mathrm{mM})$ only, or pretreated with 3-MA prior to treatment with WZY-321. The expression levels of Bcl-2 and beclin1 were assessed by western blotting following $24 \mathrm{~h}$ of incubation. DMSO, dimethyl sulfoxide; 3-MA, 3-methyladenine; PARP, poly (ADP-ribose) polymerase; $\mathrm{Bcl}-2$, apoptosis regulator Bcl-2.

Evo designed using a scaffold-hopping-based structural modification strategy. In the present study, the antiproliferative effect of WZY-321 on the growth of SHG-44 and SWO-38 cells was assessed using the CCK- 8 assay. The results indicated that the viability of SHG-44 cells treated with WZY-321 for $24 \mathrm{~h}$ was significantly decreased. This indicated the potent cytotoxicity of WZY-321, which merited further investigation for the development of novel potential anti-tumor agents. SHG-44 cells treated with WZY-321 for $24 \mathrm{~h}$ displayed marked alterations in cell morphology, exhibiting chromatin condensation, which indicated cell apoptosis. The apoptosis-associated protein $\mathrm{Bcl}-2$ was also detected in order to verify apoptosis. However, there are limitations to the present study; cells were only exposed to the drug for 24 or $48 \mathrm{~h}$, whereas exposure for $72 \mathrm{~h}$ may have enhanced the apoptotic potential of WZY-321. Furthermore, there is absence of data relating to additional apoptosis-associated proteins, including caspase 3, 7 and 9 .

There is a complex association between autophagy and apoptosis. In the treatment of GBM and anaplastic astrocytoma, autophagy mediates cell survival against anti-cancer therapies, while its excessive activation potentiates cytotoxicity and induces autophagic cell death. The inhibition of autophagy has been reported to synergize with the effect of erlotinib in inducing GBM cell death (33). By contrast, in malignant glioma cell lines, arsenic trioxide has been reported to inhibit glioma growth through the induction of autophagic cell death (34). Autophagy contributes to gefitinib-induced growth inhibition in glioma cells (35). These seemingly controversial observations result from the complex tumor microenvironment and diverse therapeutic mechanisms of anti-cancer drugs (36), all of which adds complexity to the study of autophagy-associated cancer therapies.

A previous report indicated the induction of intracellular calcium-JNK signaling-mediated autophagy and calcium-mitochondria-mediated apoptosis by Evo in glioma cells (37). These reports confirm the findings from the current study that the therapeutic potential of WZY-321 in glioma cells was associated with autophagy. However, these results require careful interpretation; the present results indicated that reduced autophagy impeded the apoptotic effects of WZY-321. However, the association between apoptosis and autophagy in SHG-44 glioma cells treated with WYZ-321 requires further investigation. Furthermore, the exact role 
of autophagy in the anti-cancer action of WZY-321 requires further pharmacological evaluation. In addition, topoisomerase 1 was previously identified as one of the targets of Evo (38). As an analog of Evo, future studies on the potential targets of WZY-321 and its mechanisms of action are required.

In conclusion, the application of WZY-321, an analog of Evo, in targeting glioma cell survival was identified. WZY-321 decreased the proliferation of SHG-44 glioma cells in a dose-dependent manner by enhancing apoptosis and inducing cell cycle arrest at the G2-M phase. Treatment with WYZ-321 significantly increased the expression of two autophagy-associated proteins, LC3 and Beclin1, in a dose-dependent manner in SHG-44 glioma cells, and this may be associated with its therapeutic potential. The results from the present study provide a possible insight into the development of novel autophagy-based therapeutic strategies for the treatment of glioblastoma.

\section{Acknowledgements}

Not applicable.

\section{Funding}

This study was supported by the National Natural Science Foundation of China (grant nos. 81672499 and 81000963), Jiangsu Province's Natural Science Foundation (grant nos. BK20141256 and BK20161318) and the Yancheng Medical Science Development Foundation (grant nos. YK2014011 and YK 2015001).

\section{Availability of data and materials}

The datasets used and-or analyzed during the current study are available from the corresponding author on reasonable request.

\section{Authors' contributions}

GS, ML and YC conceived and designed the study; GS and JG were responsible for the development and methodology of the study; GS, CZ and HS acquired the data; GS and CZ analyzed the data; GS wrote the manuscript; YC and ML supervised the study.

\section{Ethics approval and consent to participate}

Not applicable.

\section{Patient consent for publication}

Not applicable.

\section{Competing interests}

The authors declare that they have no competing interests.

\section{References}

1. Curry RC, Dahiya S, Alva Venur V, Raizer JJ and Ahluwalia MS Bevacizumab in high-grade gliomas: Past, present, and future. Expert Rev Anticancer Ther 15: 387-397, 2015.
2. Baskar R, Lee KA, Yeo R and Yeoh KW: Cancer and radiation therapy: Current advances and future directions. Int J Med Sci 9: 193-199, 2012.

3. Chang KY: Specificity protein 1-modulated superoxide dismutase 2 enhances temozolomide resistance in glioblastoma, which is independent of O6-methylguanine-DNA methyltransferase. Redox Biol 13: 655-664, 2017.

4. Kumthekar P: A phase II trial of arsenic trioxide and temozolomide in combination with radiation therapy for patients with malignant gliomas. J Neurooncol 133: 589-594, 2017.

5. Blakeley JO, Grossman SA, Mikkelsen T, Rosenfeld MR, Peereboom D, Nabors LB, Chi AS, Emmons G, Garcia Ribas I, Supko JG, et al: Phase I study of iniparib concurrent with monthly or continuous temozolomide dosing schedules in patients with newly diagnosed malignant gliomas. J Neurooncol 125: 123-131, 2015.

6. Wong ET, Timmons J, Callahan A, O'Loughlin L, Giarusso B and Alsop DC: Phase I study of low-dose metronomic temozolomide for recurrent malignant gliomas. BMC Cancer 16: 914, 2016.

7. Messaoudi K, Clavreul A and Lagarce F: Toward an effective strategy in glioblastoma treatment. Part I: Resistance mechanisms and strategies to overcome resistance of glioblastoma to temozolomide. Drug Discov Today 20: 899-905, 2015.

8. Nakada M, Kita D, Watanabe T, Hayashi Y and Hamada J: Mechanism of chemoresistance against tyrosine kinase inhibitors in malignant glioma. Brain Tumor Pathol 31: 198-207, 2014.

9. Klionsky DJ: Autophagy revisited: A conversation with Christian de Duve. Autophagy 4: 740-743, 2008.

10. Ohsumi Y: Historical landmarks of autophagy research. Cell Res 24: 9-23, 2014.

11. Liang XH, Jackson S, Seaman M, Brown K, Kempkes B, Hibshoosh H and Levine B: Induction of autophagy and inhibition of tumorigenesis bybeclin 1. Nature 402: 672-676, 1999.

12. Kabeya Y, Mizushima N, Ueno T, Yamamoto A, Kirisako T, Noda T, Kominami E, Ohsumi Y and Yoshimori T: LC3, a mammalian homologue of yeast Apg8p, is localized in autophagosome membranes after processing. EMBO J 19: 5720-5728, 2000.

13. Hart LS, Cunningham JT, Datta T, Dey S, Tameire F, Lehman SL, Qiu B, Zhang H, Cerniglia G, Bi M, et al: ER stress-mediated autophagy promotes Myc-dependent transformation and tumor growth. J Clin Invest 122: 4621-4634, 2012.

14. Aoki H, Kondo Y, Aldape K, Yamamoto A, Iwado E, Yokoyama T, Hollingsworth EF, Kobayashi R, Hess K, Shinojima N, et al: Monitoring autophagy in glioblastoma with antibody against isoform B of human microtubule-associated protein 1 light chain 3. Autophagy 4: 467-475, 2008.

15. Palumbo $\mathrm{S}$ and Comincini S: Autophagy and ionizing radiation in tumors: The 'survive or not survive' dilemma. J Cell Physiol 228: 1-8, 2013.

16. Huang X, Bai HM, Chen L, Li B and Lu YC: Reduced expression of LC3B-II and Beclin 1 in glioblastoma multiforme indicates a downregulated autophagic capacity that relates to the progression of astrocytic tumors. J Clin Nuronsci 17 : $1515-1519,2010$.

17. Miracco C, Cosci E, Oliveri G, Luzi P, Pacenti L, Monciatti I, Mannucci S, De Nisi MC, Toscano M, Malagnino V, et al: Protein and mRNA expression of autophagy gene Beclin 1 in human brain tumours. Int J Oncol 30: 429-436, 2007.

18. National Pharmacopoeia Committee: Pharmacopoeia of People's Republic of China, Part 1. The Medicine Science and Technology Press of China, Beijing, pp160-161, 2010.

19. Fan X, Zhu JY, Sun Y, Luo L, Yan J, Yang X, Yu J, Tang WQ, Ma W and Liang HP: Evodiamine inhibits zymosan-induced inflammation in vitro and in vivo: Inactivation of NF- $\mathrm{kB}$ by inhibiting I $\kappa \mathrm{B} \alpha$ phosphorylation. Inflammation 40: 1012-1027, 2017.

20. Wu WS, Chien CC, Liu KH, Chen YC and Chiu WT: Evodiamine prevents glioma growth, induces glioblastoma cell apoptosis and cell cycle arrest through JNK activation. Am J Chin Med 45: 879-899, 2017.

21. Liu LH, Xie JY, Guo WW, Wu GY, Chen ZF, Yi JY, Zhang L, Zhang ZJ and Li Z: Evodiamine activates AMPK and promotes adiponectin multimerization in 3T3-L1 adipocytes. J Asian Nat Prod Res 16: 1074-1083, 2014.

22. Zhao T, Zhang X, Zhao Y, Zhang L, Bai X, Zhang J, Zhao X, Chen L, Wang L and Cui L: Pretreatment by evodiamine is neuroprotective in cerebral ischemia: Up-regulated pAkt, pGSK $3 \beta$, down-regulated NF- $\kappa \mathrm{B}$ expression, and ameliorated BBB permeability. Neurochem Res 39: 1612-1620, 2014. 
23. Pan X, Hartley JM, Hartley JA, White KN, Wang Z and Bligh SW: Evodiamine, a dual catalytic inhibitor of type I and II topoisomerases, exhibits enhanced inhibition against camptothecin resistant cells. Phytomedicine 19: 618-624, 2012.

24. Sun HZ, Fang ZZ, Cao YF, Sun XY and Hong M: Investigation of the in vitro metabolism of evodiamine: Characterization of metabolites and involved cytochrome p450 isoforms. Phytother Res 27: 705-712, 2013.

25. Xu JY, Xu ST and Qiu YY: Evodiamine a novel class of derivatives, their preparation and use. Chinese patent 201611019652 Filed November 16, 2016.

26. Situ R, Wang HH, Wang JH, He LP, Zhu XL and Ou WF: Establishment of human brain malignant glioma cell line (SWO-38) and observation of its biologic properties. Chin J Cancer 6: 235-238, 1987 (In Chinese).

27. Pisapia DJ: The updated world health organization glioma classification: Cellular and molecular origins of adult infiltrating gliomas. Arch Pathol Lab Med 141: 1633-1645, 2017.

28. Yang L, Liang Q, Shen K, Ma L, An N, Deng W, Fei Z and Liu J: A novel class I histone deacetylase inhibitor, I-7ab, induces apoptosis and arrests cell cycle progression in human colorectal cancer cells. Biomed Pharmacother 71: 70-78, 2015.

29. Zhou X, Yue GG, Chan AM, Tsui SK, Fung KP, Sun H, Pu J and Lau CB: Eriocalyxin B, a novel autophagy inducer, exerts anti-tumor activity through the suppression of Akt-mTOR-p70S6K signaling pathway in breast cancer. Biochem Pharmacol 142: 58-70, 2017.

30. Cheng YC, Hueng DY, Huang HY, Chen JY and Chen Y: Magnolol and honokiol exert a synergistic anti-tumor effect through autophagy and apoptosis in human glioblastomas. Oncotarget 7: 29116-29130, 2016.

31. Wang XJ, Chu NY, Wang QH, Liu C, Jiang CG, Wang XY, Ikejima T and Cheng MS: Newly synthesized bis-benzimidazole derivatives exerting anti-tumor activity through induction of apoptosis and autophagy. Bioorg Med Chem Lett 22: 6297-6300, 2012.
32. Yang F, Shi L, Liang T, Ji L, Zhang G, Shen Y, Zhu F and Xu L: Anti-tumor effect of evodiamine by inducing Akt-mediated apoptosis in hepatocellular carcinoma. Biochem Biophys Res Commun 485: 54-61, 2017.

33. Eimer S, Belaud-Rotureau MA, Airiau K, Jeanneteau M, Laharanne E, Véron N, Vital A, Loiseau H, Merlio JP and Belloc F: Autophagy inhibition cooperates with erlotinib to induce glioblastoma cell death. Cancer Biol Ther 11: 1017-1027, 2011.

34. Kanzawa T, Zhang L, Xiao L, Germano IM, Kondo Y and Kondo S: Arsenic trioxide induces autophagic cell death in malignant glioma cells by upregulation of mitochondrial cell death protein BNIP3. Oncogene 24: 980-991, 2005.

35. Chang CY, Kuan YH, Ou YC, Li JR, Wu CC, Pan PH, Chen WY, Huang HY and Chen CJ: Autophagy contributes to gefitinib-induced glioma cell growth inhibition. Exp Cell Res 327: 102-112, 2014.

36. Grandér D and Panaretakis T: Autophagy: Cancer therapy's friend or foe? Future Med Chem 2: 285-297, 2010.

37. Liu AJ, Wang SH, Chen KC, Kuei HP, Shih YL, Hou SY, Chiu WT, Hsiao SH and Shih CM: Evodiamine, a plant alkaloid, induces calcium-JNK-mediated autophagy and calcium-mitochondria-mediated apoptosis in human glioblastoma cells. Chem Biol Interact 205: 20-28, 2013.

38. Dong G, Sheng C, Wang S, Miao Z, Yao J and Zhang W Selection of evodiamine as a novel topoisomerase I inhibitor by structure-based virtual screening and hit optimization of evodiamine derivatives as antitumor agents. J Med Chem 53: 7521-7531, 2010 